Volume 133, Number 11, Pages 3271-3280

S 0002-9939(05)07937-2

Article electronically published on May 2, 2005

\title{
GRÜSS TYPE INEQUALITIES IN INNER PRODUCT MODULES
}

\author{
DIJANA ILIŠEVIĆ AND SANJA VAROŠANEC
}

(Communicated by David R. Larson)

\begin{abstract}
In this paper we give some properties of a generalized inner product in modules over $\mathrm{H}^{*}$-algebras and $\mathrm{C}^{*}$-algebras and we obtain inequalities of Grüss type.
\end{abstract}

\section{INTRODUCTION}

One of the earliest results which gave estimates for the Chebyshev difference

$$
T(f, g)=\frac{1}{b-a} \int_{a}^{b} f(x) g(x) d x-\frac{1}{(b-a)^{2}} \int_{a}^{b} f(x) d x \int_{a}^{b} g(x) d x, \quad f, g \in L[a, b],
$$

was the Grüss inequality given in the following theorem ([5], see also [9, pp. 295$310])$.

Theorem 1.1. Let $f$ and $g$ be real functions defined and integrable on $[a, b] \subset \mathbb{R}$. If there exist real constants $\varphi, \phi, \gamma, \Gamma$ such that

$$
\varphi \leq f(x) \leq \phi, \quad \gamma \leq g(x) \leq \Gamma, \quad \text { for } \quad x \in[a, b],
$$

then

$$
T(f, g) \leq \frac{1}{4}(\phi-\varphi)(\Gamma-\gamma)
$$

The constant $\frac{1}{4}$ is the best possible.

This inequality was investigated and generalized by a number of mathematicians. These investigations of the Grüss and related inequalities have been stimulated by their applications in different areas of applied mathematics, such as in perturbed quadrature rules ([1, [2]) and in the approximation of integral transforms ([4, 6] and references therein). For further reading about related results we refer to the monograph [9] pp. 295-310]. In the last decade some papers about Grüss type inequalities in real and complex inner product spaces have appeared. Here we mention papers such as [3] and [15].

Received by the editors June 11, 2004.

2000 Mathematics Subject Classification. Primary 46L08, 46H25; Secondary 46CXX, 26D99.

Key words and phrases. Generalized inner product, inner product $\mathrm{H}^{*}$-module, inner product $\mathrm{C}^{*}$-module, Cauchy-Schwarz inequality, Grüss inequality.

(C) 2005 American Mathematical Society
public domain 28 years from publication Reverts to public domain 28 years from publication 
The basic result from these papers is the following theorem ([3]):

Theorem 1.2. Let $(H,(.,)$.$) be an inner product space over \mathbb{K}(\mathbb{K}=\mathbb{R}, \mathbb{C})$ and $e \in H,\|e\|=1$. If $\varphi, \phi, \gamma, \Gamma \in \mathbb{K}$ and $x, y$ are vectors in $H$ such that conditions

$$
\left\|x-\frac{\phi+\varphi}{2} e\right\| \leq \frac{1}{2}|\phi-\varphi|, \quad\left\|y-\frac{\Gamma+\gamma}{2} e\right\| \leq \frac{1}{2}|\Gamma-\gamma|
$$

hold, then we have the inequality

$$
|(x, y)-(x, e)(e, y)| \leq \frac{1}{4}|\phi-\varphi||\Gamma-\gamma|
$$

The constant $\frac{1}{4}$ is the best possible in the sense that it cannot be replaced by a smaller one.

In this paper we give inequalities of the Grüss type in certain abstract structures called inner product modules. The second section contains preliminaries. In the third section we describe a new generalized semi-inner product generated by the generalized inner product which is the first-defined product in an inner product module. In the fourth and the fifth sections Grüss type inequalities and their refinement in inner product $\mathrm{H}^{*}$-modules and inner product $\mathrm{C}^{*}$-modules are proved.

\section{Preliminaries}

An inner product module arises as a generalization of a complex inner product space when the complex field is replaced by a $\mathrm{C}^{*}$-algebra or a proper $\mathrm{H}^{*}$-algebra. The idea of replacing the complex numbers by the elements of a $\mathrm{C}^{*}$-algebra first appeared in the work of Kaplansky $\left([7)\right.$ and by the elements of a proper $\mathrm{H}^{*}$-algebra in the work of Saworotnow $([12])$.

A proper $\mathrm{H}^{*}$-algebra is a complex Banach $*$-algebra $(A,\|\|$.$) whose underlying$ Banach space is a Hilbert space with respect to the inner product $\langle.,$.$\rangle satisfying$ $\langle a b, c\rangle=\left\langle b, a^{*} c\right\rangle$ and $\langle b a, c\rangle=\left\langle b, c a^{*}\right\rangle$ for all $a, b, c \in A$. A C*-algebra is a complex Banach $*$-algebra $(A,\|\|$.$) such that \left\|a^{*} a\right\|=\|a\|^{2}$ for every $a \in A$.

If $A$ is a proper $\mathrm{H}^{*}$-algebra or a $\mathrm{C}^{*}$-algebra and $a \in A$ is such that $A a=0$ or $a A=0$, then $a=0$.

An element $a$ in a proper $\mathrm{H}^{*}$-algebra $A$ is called positive $(a \geq 0)$ if $\langle a x, x\rangle \geq 0$ for every $x \in A$. Every positive element $a$ in a proper $\mathrm{H}^{*}$-algebra is self-adjoint (that is, $\left.a^{*}=a\right)$. An element $a$ in a $\mathrm{C}^{*}$-algebra $A$ is called positive $(a \geq 0)$ if it is self-adjoint and has positive spectrum. An element $a^{*} a$ is positive for every $a \in A$, in both structures.

The trace-class associated with a proper $\mathrm{H}^{*}$-algebra $A$ is defined as the set $\tau(A)=$ $\{a b: a, b \in A\}$. It is a self-adjoint two-sided ideal of $A$ which is dense in $A$. There is a positive linear functional tr on $\tau(A)$, called trace, such that $\operatorname{tr}(a b)=\operatorname{tr}(b a)=$ $\left\langle a, b^{*}\right\rangle=\left\langle b, a^{*}\right\rangle$ for all $a, b \in A$. In particular, $\operatorname{tr}\left(a^{*} a\right)=\|a\|^{2}$ for every $a \in A$.

If $A$ is a proper $\mathrm{H}^{*}$-algebra, for every positive $a \in \tau(A)$ there exists a unique positive $b \in A$ such that $b^{2}=a$. Such a $b$ is denoted by $a^{1 / 2}$, and it is called the square root of $a$. If $A$ is a $\mathrm{C}^{*}$-algebra, the square root of $a$ exists for every positive $a \in A$. Especially, in both cases, for every $a \in A$ there exists the square root of $a^{*} a$ which is denoted by $|a|$ and called the absolute value of $a$.

The trace-class is a Banach *-algebra with respect to the norm $\tau($.$) defined$ by $\tau(a)=\operatorname{tr}(|a|)$. Let us mention that $|\operatorname{tr}(a)| \leq \tau(a)$ and $\|a\| \leq \tau(a)$ for every $a \in \tau(A)$. More details concerning the trace-class can be found in [13] and [14]. 
Let $A$ be a proper $\mathrm{H}^{*}$-algebra or a $\mathrm{C}^{*}$-algebra. A semi-inner product module over $A$ is a right module $H$ over $A$ together with a generalized semi-inner product, that is, with a mapping [.,.] on $H \times H$, which is $\tau(A)$-valued if $A$ is a proper $\mathrm{H}^{*}$-algebra, or $A$-valued if $A$ is a $\mathrm{C}^{*}$-algebra, having the following properties:

(i) $[f, g+h]=[f, g]+[f, h]$ for all $f, g, h \in H$,

(ii) $[f, g a]=[f, g] a$ for all $f, g \in H$ and $a \in A$,

(iii) $[f, g]^{*}=[g, f]$ for all $f, g \in H$,

(iv) $[f, f] \geq 0$ for every $f \in H$.

Since $[f, f]$ is a positive element in $\tau(A)$ if $A$ is a proper $\mathrm{H}^{*}$-algebra, and in $A$ if $A$ is a $\mathrm{C}^{*}$-algebra, there is the square root of $[f, f]$ which is denoted by $|f|$ and called the absolute value of $f$.

If, in addition, $[f, f]=0$ implies $f=0$, then $[.,$.$] is called a generalized inner$ product and $H$ is called an inner product module over $A$. We shall say that $H$ is a (semi-)inner product $\mathrm{H}^{*}$-module if it is a (semi-)inner product module over a proper $\mathrm{H}^{*}$-algebra, and that $H$ is a (semi-)inner product $\mathrm{C}^{*}$-module if it is a (semi-)inner product module over a $\mathrm{C}^{*}$-algebra.

As we can see, an inner product module obeys the same axioms as an ordinary inner product space, except that the inner product takes values in a more general structure than in the field of complex numbers.

If $H$ is a semi-inner product $\mathrm{C}^{*}$-module, then the following Cauchy-Schwarz inequality holds:

$$
\|[f, g]\|^{2} \leq\|[f, f]\| \cdot\|[g, g]\| \quad(f, g \in H)
$$

(e.g. [8 Proposition 1.1] together with [11, Theorem 2.2.5.(3)]).

If $H$ is a semi-inner product $\mathrm{H}^{*}$-module, then there are two forms of the CauchySchwarz inequality:

$$
|\operatorname{tr}([f, g])|^{2} \leq \operatorname{tr}([f, f]) \cdot \operatorname{tr}([g, g]) \quad(f, g \in H)
$$

(the weak Cauchy-Schwarz inequality), and

$$
\tau([f, g])^{2} \leq \operatorname{tr}([f, f]) \cdot \operatorname{tr}([g, g]) \quad(f, g \in H)
$$

(the strong Cauchy-Schwarz inequality). In his pioneering paper 12, Saworotnow proved the strong Cauchy-Schwarz inequality, but the set of axioms he was dealing with was richer than in the definition of a semi-inner product $\mathrm{H}^{*}$-module. The direct proof of the strong Cauchy-Schwarz inequality for a semi-inner product $\mathrm{H}^{*}$ module can be found in [10].

Lemma 2.1. Let $A$ be a proper $H^{*}$-algebra or a $C^{*}$-algebra, and let $H$ be a semiinner product module over $A$. For all $f, g \in H$,

$$
[f, g]+[g, f] \leq \frac{1}{2}|f+g|^{2} .
$$

Proof.

$|f+g|^{2}-2([f, g]+[g, f])=[f+g, f+g]-2([f, g]+[g, f])=[f-g, f-g] \geq 0$. 
Every proper $\mathrm{H}^{*}$-algebra $A$ and every $\mathrm{C}^{*}$-algebra $A$ is an inner product module over itself with respect to the generalized inner product given by $[a, b]=a^{*} b$ for all $a, b \in A$. Therefore, an immediate consequence of Lemma 2.1 is:

Corollary 2.2. Let $A$ be a proper $H^{*}$-algebra or a $C^{*}$-algebra. For all $a, b \in A$,

$$
a^{*} b+b^{*} a \leq \frac{1}{2}|a+b|^{2}
$$

If $A$ is a proper $\mathrm{H}^{*}$-algebra or a $\mathrm{C}^{*}$-algebra, then $p \in A$ is called a projection if it is non-zero and $p^{*}=p=p^{2}$. Let us emphasize that $\|p\|=1$ if $A$ is a $\mathrm{C}^{*}$-algebra, but if $A$ is a proper $\mathrm{H}^{*}$-algebra, then $\|p\|^{2}=\operatorname{tr}(p)$, which is not equal to 1 in general.

Definition 2.3. Let $A$ be a proper $\mathrm{H}^{*}$-algebra or a $\mathrm{C}^{*}$-algebra, and let $(H,[.,]$.$) be$ a semi-inner product module over $A$. An element $h \in H$ is called a lifted projection if $|h|$ is a projection in $A$.

In the following lemma a useful characterization of lifted projections in inner product modules is given.

Lemma 2.4. Let $(H,[.,]$.$) be an inner product H^{*}$-module or an inner product $C^{*}$-module and let $h \in H$. Then $h$ is a lifted projection if and only if $h|h|=h$.

Proof. The equality

$$
\begin{aligned}
& |h| h|-h|^{2}=[h|h|-h, h|h|-h] \\
= & {[h|h|, h|h|]-[h, h|h|]-[h|h|, h]+[h, h] } \\
= & |h|[h, h]|h|-[h, h]|h|-|h|[h, h]+[h, h] \\
= & |h| \cdot|h|^{2} \cdot|h|-|h|^{2} \cdot|h|-|h| \cdot|h|^{2}+|h|^{2}=\left.|| h\right|^{2}-\left.|h|\right|^{2}
\end{aligned}
$$

implies that $h|h|-h=0$ if and only if $|h|^{2}=|h|$.

Although there are inner product $\mathrm{H}^{*}$-modules and inner product $\mathrm{C}^{*}$-modules which cannot be equipped with the structure of a complex vector space compatible with the structure of $A$, Lemma 2.4 enables us to define $\alpha h:=h(\alpha|h|)$ for every $\alpha \in \mathbb{C}$ and every lifted projection $h \in H$.

The following technical lemma will be needed later on.

Lemma 2.5. Let $A$ be a proper $H^{*}$-algebra or a $C^{*}$-algebra. Let $(H,[.,]$.$) be an$ inner product module over $A$, and let $h \in H$ be a lifted projection. For all $f \in H$ and $\alpha, \beta \in \mathbb{C}$,

$$
0 \leq|f|^{2}-[f, h][h, f] \leq\left|f-\frac{\alpha+\beta}{2} h\right|^{2}
$$

Proof. First we have

$$
\begin{aligned}
& |f|^{2}-[f, h][h, f]=[f, f]-[f, h][h, f] \\
= & {[f, f]-[h, f]^{*}[h, f]-[f, h][h, f]+[f, h][h, f] } \\
= & {[f, f]-[h, f]^{*}[h, f]-[f, h][h, f]+[f, h|h|][h|h|, f] } \\
= & {[f, f]-[h, f]^{*}[h, f]-[f, h][h, f]+[f, h]|h|^{2}[h, f] } \\
= & {[f, f]-[h, f]^{*}[h, f]-[f, h][h, f]+[h, f]^{*}[h, h][h, f] } \\
= & {[f-h[h, f], f-h[h, f]] \geq 0 . }
\end{aligned}
$$


If we denote

$$
x=[\alpha h-f, f-\beta h], \quad y=(\alpha|h|-[h, f])^{*}([h, f]-\beta|h|),
$$

then

$$
\begin{aligned}
& y-x=(\bar{\alpha}|h|-[f, h])([h, f]-\beta|h|)-[\alpha h-f, f-\beta h] \\
= & \bar{\alpha}|h|[h, f]-[f, h][h, f]-\bar{\alpha} \beta|h|^{2}+\beta[f, h]|h|-[\alpha h, f]+[f, f] \\
& +[\alpha h, \beta h]-[f, \beta h] \\
= & {[\alpha h, f]-[f, h][h, f]-\bar{\alpha} \beta|h|^{2}+[f, \beta h]-[\alpha h, f]+|f|^{2}+[\alpha h, \beta h]-[f, \beta h] } \\
= & |f|^{2}-[f, h][h, f] .
\end{aligned}
$$

Applying Corollary 2.2 on $\alpha|h|-[h, f]$ and $[h, f]-\beta|h|$, we get

$$
\begin{aligned}
& (\alpha|h|-[h, f])^{*}([h, f]-\beta|h|)+([h, f]-\beta|h|)^{*}(\alpha|h|-[h, f]) \\
\leq & \frac{1}{2}|(\alpha|h|-[h, f])+([h, f]-\beta|h|)|^{2}=\frac{1}{2}|\alpha-\beta|^{2}|h|,
\end{aligned}
$$

that is,

Therefore,

$$
y+y^{*} \leq \frac{1}{2}|\alpha-\beta|^{2}|h|
$$

$$
\begin{aligned}
& |f|^{2}-[f, h][h, f]=\frac{1}{2}\left((y-x)+(y-x)^{*}\right)=\frac{1}{2}\left(y+y^{*}\right)-\frac{1}{2}\left(x+x^{*}\right) \\
\leq & \frac{1}{2}\left(\frac{1}{2}|\alpha-\beta|^{2}|h|\right)-\frac{1}{2}\left(x+x^{*}\right)=\frac{1}{4}|\alpha-\beta|^{2}|h|-\frac{1}{2}\left(x+x^{*}\right) \\
= & \frac{1}{4}|\alpha-\beta|^{2}|h|-\frac{1}{2}([\alpha h-f, f-\beta h]+[f-\beta h, \alpha h-f]) \\
= & \frac{1}{4}|\alpha-\beta|^{2}|h|^{2}-\left(\frac{\overline{\alpha+\beta}}{2}[h, f]+\frac{\alpha+\beta}{2}[f, h]-|f|^{2}-\frac{\bar{\alpha} \beta+\alpha \bar{\beta}}{2}|h|^{2}\right) \\
= & |f|^{2}-\frac{\alpha+\beta}{2}[f, h]-\frac{\overline{\alpha+\beta}}{2}[h, f]+\frac{|\alpha+\beta|^{2}}{4}|h|^{2} \\
= & {\left[f-\frac{\alpha+\beta}{2} h, f-\frac{\alpha+\beta}{2} h\right]=\left|f-\frac{\alpha+\beta}{2} h\right|^{2} . }
\end{aligned}
$$

\section{On a Generalized SEMi-INNER PRODUCt GENERATEd by $[.,$.}

Definition 3.1. Let $(H,[.,]$.$) be an inner product \mathrm{H}^{*}$-module or an inner product $\mathrm{C}^{*}$-module over $A$ and let $h \in H$ be a lifted projection. The mapping $\llbracket ., . \rrbracket_{h}$ is defined on $H \times H$ by

$$
\llbracket f, g \rrbracket_{h}=[f, g]-[f, h][h, g] .
$$

Note that the mapping $\llbracket ., . \rrbracket_{h}$ is $\tau(A)$-valued if $A$ is a proper $\mathrm{H}^{*}$-algebra, and $A$-valued if $A$ is a $\mathrm{C}^{*}$-algebra.

As simple consequences of the properties of the generalized inner product $[.,$. the following properties are valid:

(i) $\llbracket f, g+k \rrbracket_{h}=\llbracket f, g \rrbracket_{h}+\llbracket f, k \rrbracket_{h}$ for all $f, g, k \in H$,

(ii) $\llbracket f, g a \rrbracket_{h}=\llbracket f, g \rrbracket_{h} a$ for all $f, g \in H$ and $a \in A$,

(iii) $\llbracket f, g \rrbracket_{h}^{*}=\llbracket g, f \rrbracket_{h}$ for all $f, g \in H$. 
From Lemma 2.5 we obtain the positivity of $\llbracket ., \rrbracket_{h}$ :

(iv) $\llbracket f, f \rrbracket_{h} \geq 0$ for every $f \in H$.

Hence, we have proved the following proposition.

Proposition 3.2. Let $(H,[.,]$.$) be an inner product H^{*}$-module or an inner product $C^{*}$-module and let $h$ be a lifted projection in $H$. The function $\llbracket ., . \rrbracket_{h}$ defined by

$$
\llbracket f, g \rrbracket_{h}=[f, g]-[f, h][h, g]
$$

is a generalized semi-inner product on $H$.

Theorem 3.3. Let $(H,[.,]$.$) be an inner product H^{*}$-module and let $h$ be a lifted projection in $H$. Then for any $f, g \in H$ the following inequality holds:

$$
\tau([f, g]-[f, h][h, g])^{2} \leq\left(\||f|\|^{2}-\|[h, f]\|^{2}\right) \cdot\left(\||g|\|^{2}-\|[h, g]\|^{2}\right) .
$$

Proof. Using the strong Cauchy-Schwarz inequality for $\llbracket ., . \rrbracket_{h}$ we have

$$
\begin{aligned}
& \tau([f, g]-[f, h][h, g])^{2}=\tau\left(\llbracket f, g \rrbracket_{h}\right)^{2} \leq \operatorname{tr}\left(\llbracket f, f \rrbracket_{h}\right) \cdot \operatorname{tr}\left(\llbracket g, g \rrbracket_{h}\right) \\
= & \left(\operatorname{tr}\left(|f|^{2}\right)-\operatorname{tr}\left([h, f]^{*}[h, f]\right)\right) \cdot\left(\operatorname{tr}\left(|g|^{2}\right)-\operatorname{tr}\left([h, g]^{*}[h, g]\right)\right) \\
= & \left(\||f|\|^{2}-\|[h, f]\|^{2}\right) \cdot\left(\||| g \mid\|^{2}-\|[h, g]\|^{2}\right) .
\end{aligned}
$$

Theorem 3.4. Let $(H,[.,]$.$) be an inner product C^{*}$-module and let $h$ be a lifted projection in $H$. Then for any $f, g \in H$ the following inequality holds:

$$
\|[f, g]-[f, h][h, g]\|^{2} \leq\left\||f|^{2}-[f, h][h, f]\right\| \cdot\left\||g|^{2}-[g, h][h, g]\right\| .
$$

Proof. Using Cauchy-Schwarz inequality for $\llbracket ., . \rrbracket_{h}$ we have

$$
\begin{aligned}
& \|[f, g]-[f, h][h, g]\|^{2}=\left\|\llbracket f, g \rrbracket_{h}\right\|^{2} \leq\left\|\llbracket f, f \rrbracket_{h}\right\| \cdot\left\|\llbracket g, g \rrbracket_{h}\right\| \\
= & \left\||f|^{2}-[f, h][h, f]\right\| \cdot\left\||g|^{2}-[g, h][h, g]\right\| .
\end{aligned}
$$

\section{Grüss inequality In INNER PROduCt H*-MOdules}

Theorem 4.1. Let $A$ be a proper $H^{*}$-algebra. Let $(H,[.,]$.$) be an inner product$ module over $A$ and let $h \in H$ be a lifted projection. If $\varphi, \phi, \gamma, \Gamma \in \mathbb{C}$ and $f, g \in H$ are such that the assumptions

$$
\left\|\left|f-\frac{\phi+\varphi}{2} h\right|\right\| \leq \frac{1}{2}|\phi-\varphi| \cdot\||h|\|, \quad\left\|\left|g-\frac{\Gamma+\gamma}{2} h\right|\right\| \leq \frac{1}{2}|\Gamma-\gamma| \cdot\||h|\|
$$

hold, then we have the inequality

$$
\begin{aligned}
\tau([f, g] & -[f, h][h, g]) \leq \frac{1}{4}\left|\phi-\varphi\left\|\Gamma-\gamma\left|\||h|\|^{2}\right.\right.\right. \\
& -\left(\frac{1}{4}|\phi-\varphi|^{2} \cdot\||h|\|^{2}-\|\left.\left|f-\frac{\phi+\varphi}{2} h\right|\right|^{2}\right)^{\frac{1}{2}} \\
& \times\left(\frac{1}{4}|\Gamma-\gamma|^{2} \cdot\||h|\|^{2}-\|\left.\left|g-\frac{\Gamma+\gamma}{2} h\right|\right|^{2}\right)^{\frac{1}{2}} .
\end{aligned}
$$

The constant $\frac{1}{4}$ is the best possible. 
Proof. From Lemma 2.5 we have

$$
|f|^{2}-[f, h][h, f] \leq\left|f-\frac{\phi+\varphi}{2} h\right|^{2} .
$$

This implies

$$
\operatorname{tr}\left(|f|^{2}-[f, h][h, f]\right) \leq \operatorname{tr}\left(\left|f-\frac{\phi+\varphi}{2} h\right|^{2}\right)
$$

that is,

$$
\||f|\|^{2}-\|[h, f]\|^{2} \leq\left\|\left|f-\frac{\phi+\varphi}{2} h\right|\right\|^{2}
$$

Analogously,

$$
\||g|\|^{2}-\|[h, g]\|^{2} \leq\left\|\left|g-\frac{\Gamma+\gamma}{2} h\right|\right\|^{2} .
$$

After multiplying (4.3) and (4.4), we obtain

$$
\left(\||f|\|^{2}-\|[h, f]\|^{2}\right) \cdot\left(\||g|\|^{2}-\|[h, g]\|^{2}\right) \leq\left\|\left|f-\frac{\phi+\varphi}{2} h\right|\right\|^{2} \cdot\left\|\left|g-\frac{\Gamma+\gamma}{2} h\right|\right\|^{2} .
$$

Applying the inequality $\left(m^{2}-n^{2}\right)\left(p^{2}-q^{2}\right) \leq(m p-n q)^{2}([9$ p. 117]) on

$$
\begin{gathered}
m=\frac{1}{2}|\phi-\varphi|\||h|\|, \quad n=\left(\frac{1}{4}|\phi-\varphi|^{2} \cdot\||h|\|^{2}-\left\|\left|f-\frac{\phi+\varphi}{2} h\right|\right\|^{2}\right)^{\frac{1}{2}}, \\
p=\frac{1}{2}|\Gamma-\gamma|\||h|\|, \quad q=\left(\frac{1}{4}|\Gamma-\gamma|^{2} \cdot\||h|\|^{2}-\left\|\left|g-\frac{\Gamma+\gamma}{2} h\right|\right\|^{2}\right)^{\frac{1}{2}},
\end{gathered}
$$

we get

$$
\begin{gathered}
\left\|\left|f-\frac{\phi+\varphi}{2} h\right|\right\|^{2} \cdot\left\|\left|g-\frac{\Gamma+\gamma}{2} h\right|\right\|^{2} \leq\left(\frac{1}{4}|\phi-\varphi||\Gamma-\gamma|\||h|\|^{2}\right. \\
-\left(\frac{1}{4}|\phi-\varphi|^{2} \cdot\||h|\|^{2}-\left\|\left|f-\frac{\phi+\varphi}{2} h\right|\right\|^{2}\right)^{\frac{1}{2}} \\
\left.\quad \times\left(\frac{1}{4}|\Gamma-\gamma|^{2} \cdot\||h|\|^{2}-\left\|\left|g-\frac{\Gamma+\gamma}{2} h\right|\right\|^{2}\right)^{\frac{1}{2}}\right)^{2} .
\end{gathered}
$$

It remains to compare the inequalities (3.1), (4.5) and 4.6).

If $l$ is a non-zero element in $H$ such that $[l, h]=0$, then there exists $a \in A$ satisfying $|l| a \neq 0$. If we define $\lambda=\frac{\||h|\|}{\||l| a\|}$ and $k=l(\lambda a)$, then $[k, h]=0$ and $\||k|\|=\||h|\|$. For

$$
f=\frac{|\phi-\varphi|}{2} k+\frac{\phi+\varphi}{2} h, \quad g=\frac{|\Gamma-\gamma|}{2} k+\frac{\Gamma+\gamma}{2} h
$$

the assumptions (4.1) are fulfilled and (4.2) becomes an equality. Note that the second summand on the right side in (4.2) vanishes for chosen $f$ and $g$. 
Corollary 4.2. Let $A$ be a proper $H^{*}$-algebra. Let $(H,[.,]$.$) be an inner product$ module over $A$ and let $h \in H$ be a lifted projection. If $\varphi, \phi, \gamma, \Gamma \in \mathbb{C}$ and $f, g \in H$ are such that the assumptions (4.1) hold, then

$$
\tau([f, g]-[f, h][h, g]) \leq \frac{1}{4}\left|\phi-\varphi\left\|\Gamma-\gamma\left|\||h|\|^{2} .\right.\right.\right.
$$

The constant $\frac{1}{4}$ is the best possible.

\section{Grüss inequality in inner Product $\mathrm{C}^{*}$-modules}

Theorem 5.1. Let $A$ be a $C^{*}$-algebra. Let $(H,[.,]$.$) be an inner product module$ over $A$ and let $h \in H$ be a lifted projection. If $\varphi, \phi, \gamma, \Gamma \in \mathbb{C}$ and $f, g \in H$ are such that the assumptions

$$
\left\|\left|f-\frac{\phi+\varphi}{2} h\right|\right\| \leq \frac{1}{2}|\phi-\varphi|, \quad\left\|\left|g-\frac{\Gamma+\gamma}{2} h\right|\right\| \leq \frac{1}{2}|\Gamma-\gamma|
$$

hold, then we have the inequality

$$
\begin{aligned}
& \|[f, g]-[f, h][h, g]\| \leq \frac{1}{4}|\phi-\varphi||\Gamma-\gamma| \\
& -\left(\frac{1}{4}|\phi-\varphi|^{2}-\left\|\left|f-\frac{\phi+\varphi}{2} h\right|\right\|^{2}\right)^{\frac{1}{2}}\left(\frac{1}{4}|\Gamma-\gamma|^{2}-\left\|\left|g-\frac{\Gamma+\gamma}{2} h\right|\right\|^{2}\right)^{\frac{1}{2}} .
\end{aligned}
$$

The constant $\frac{1}{4}$ is the best possible.

Proof. According to Lemma 2.5

$$
0 \leq|f|^{2}-[f, h][h, f] \leq\left|f-\frac{\phi+\varphi}{2} h\right|^{2} .
$$

If we apply [11, Theorem 2.2.5.(3)], we get

$$
\left\||f|^{2}-[f, h][h, f]\right\| \leq\left\|\left|f-\frac{\phi+\varphi}{2} h\right|^{2}\right\|,
$$

that is,

$$
\left\||f|^{2}-[f, h][h, f]\right\| \leq\left\|\left|f-\frac{\phi+\varphi}{2} h\right|\right\|^{2} .
$$

In a similar way we conclude that

$$
\left\||g|^{2}-[g, h][h, g]\right\| \leq\left\|\left|g-\frac{\Gamma+\gamma}{2} h\right|\right\|^{2} .
$$

From (5.3) and (5.4), the inequality

$$
\left\||f|^{2}-[f, h][h, f]\right\| \cdot\left\||g|^{2}-[g, h][h, g]\right\| \leq\left\|\left|f-\frac{\phi+\varphi}{2} h\right|\right\|^{2} \cdot\left\|\left|g-\frac{\Gamma+\gamma}{2} h\right|\right\|^{2}
$$

follows. As in the proof of Theorem 4.1, we get (4.6). Let us emphasize that $\||h|\|=1$ in our case. Finally, compare the inequalities (3.2), (5.5) and (4.6), taking into account that $\||h|\|=1$. 
The equality in (5.2) holds for the same $f, g \in H$ for which we get the equality in (4.2). Again the second summand on the right side vanishes for such $f$ and $g$.

Corollary 5.2. Let $A$ be a $C^{*}$-algebra. Let $(H,[.,]$.$) be an inner product module$ over $A$ and let $h \in H$ be a lifted projection. If $\varphi, \phi, \gamma, \Gamma \in \mathbb{C}$ and $f, g \in H$ are such that the assumptions (5.1) hold, then

$$
\|[f, g]-[f, h][h, g]\| \leq \frac{1}{4}|\phi-\varphi||\Gamma-\gamma|
$$

The constant $\frac{1}{4}$ is the best possible.

Remark 5.3. If $A$ is the $\mathrm{C}^{*}$-algebra of complex numbers, the related result can be found in [3].

Remark. It would be interesting, if possible, to obtain Grüss type inequalities in inner product $\mathrm{H}^{*}$-modules and inner product $\mathrm{C}^{*}$-modules inside the positive cone of the underlying algebra, that is, to obtain analogous results dropping the norms.

\section{ACKNOWLEDGEMENTS}

We express our thanks to the referee for his useful comments.

\section{REFERENCES}

1. P. Cerone, S. S. Dragomir, A refinement of the Grüss inequality and applications, RGMIA Res. Rep. Coll. 5 (2)(2002). Article 14 [http://rgmia.vu.edu.au/v5n2.html]

2. X.-L. Cheng, J. Sun, Note on the perturbed trapezoid inequality, J. Inequal. Pure \& Appl. Math. 3(2002). No. 2, Article 29 [http://jipam.vu.edu.au/v3n2/046_01.html] MR 1906398 $(2003 \mathrm{c}: 26027)$

3. S. S. Dragomir, A generalization of Grüss's inequality in inner product spaces and applications, J. Math. Anal. Appl. 237 (1999), 74-82. MR.1708162 (2000h:26038)

4. S. S. Dragomir, A. Kalam, An approximation of the Fourier Sine Transform via Grüss type inequalities and applications for electrical circuits, J. KSIAM 63 (1)(2002), 33-45.

5. G. Grüss, Über das Maximum des absoluten Betrages von $\frac{1}{b-a} \int_{a}^{b} f(x) g(x) d x-$ $\frac{1}{(b-a)^{2}} \int_{a}^{b} f(x) d x \int_{a}^{b} g(x) d x$, Math. Z. 39 (1934), 215-226.

6. G. Hanna, S. S. Dragomir, J. Roumeliotis, An approximation for the finite-Fourier transform of two independent variables, Proc. 4th Int. Conf. on Modelling and Simulation, Victoria University, Melbourne, 2002, 375-380.

7. I. Kaplansky, Modules over operator algebras, Amer. J. Math. 75 (1953), 839-858. MR0058137 $(15: 327 \mathrm{f})$

8. E. C. Lance, Hilbert $C^{*}$-modules. A toolkit for operator algebraists, London Math. Soc. Lecture Series, 210. Cambridge University Press, Cambridge, 1995. MR.1325694 (96k:46100)

9. D. S. Mitrinović, J. E. Pečarić, A. M. Fink, Classical and new inequalities in analysis, Kluwer Acad. Publ. Dordrecht, 1993. MR 1220224 (94c:00004)

10. L. Molnár, A note on the strong Schwarz inequality in Hilbert A-modules, Publ. Math. Debrecen 40 (1992), 323-325. MR1181377 (93i:46094)

11. G. J. Murphy, $C^{*}$-algebras and operator theory, Academic Press, Inc., Boston, 1990. MR 1074574 (91m:46084)

12. P. P. Saworotnow, A generalized Hilbert space, Duke Math. J. 35 (1968), 191-197. MR0227749 $(37: 3333)$

13. P. P. Saworotnow, J. C. Friedell, Trace-class for an arbitrary $H^{*}$-algebra, Proc. Amer. Math. Soc. 26 (1970), 95-100. MR0267402 (42:2304) 
14. P. P. Saworotnow, Trace-class and centralizers of an $H^{*}$-algebra, Proc. Amer. Math. Soc. 26 (1970), 101-104. MR0267403 (42:2305)

15. N. Ujević, A new generalization of Grüss inequality in inner product spaces, Math. Inequal. \& Appl. 6, No 4, (2003), 617-623. MR2013524

Department of Mathematics, University of Zagreb, Bijenička 30, P. O. Box 335, 10002 ZAGReb, CROATIA

E-mail address: ilisevic@math.hr

Department of Mathematics, University of Zagreb, Bijenička 30, P. O. Box 335, 10002 Zagreb, Croatia

E-mail address: varosans@math.hr 